\title{
Case Series of Hemolysis in Neonate Born to Mothers with History of SARS-CoV-2 Infection During Pregnancy
}

\author{
Elena Itriago ${ }^{1}$, Krithika Lingappan ${ }^{1}$, Jun Teruya ${ }^{1}$, and Daniela Dinu ${ }^{1}$ \\ ${ }^{1}$ Baylor College of Medicine
}

October 26, 2020

\begin{abstract}
Hemolytic anemia has been associated with SARS-CoV-2 infection in adult and pediatric patients, we report a case series of preterm newborns with hemolytic anemia whose mothers had a history of SARS-CoV-2 infection. In two of the cases maternal infection occurred early in pregnancy and the newborns had a reactive SARS-CoV-2 antibody test. Hemolysis and coagulopathy improved with the use of washed blood products and prothrombin complex concentrate. Our cases highlight the need for increased awareness of a possible association between hemolysis and exposure to SARS-CoV-2 infection during intrauterine life.
\end{abstract}

\section{Introduction}

We describe three neonates born to mothers with history of SARS-CoV-2 infection during pregnancy who developed hemolytic anemia.

\section{Case 1}

A 29-weeks baby girl born to a mother with SARS-CoV-2 pneumonia and acute respiratory failure (Table 1) was delivered due to non-reassuring fetal heart tones. She had an uneventful hospital course until day of life (DOL) 17 when she developed abdominal distension, hypotension, and bloody stools. She underwent a sepsis evaluation, and antibiotics were started. The initial abdomen X-ray was negative for pneumatosis, and an abdominal ultrasound was negative for volvulus or malrotation. She received red blood cells (RBC) transfusion for hemoglobin of $10.1 \mathrm{~g} / \mathrm{dL}$. On DOL 18, her status deteriorated with respiratory failure requiring intubation, refractory hypotension requiring vasopressors, persistent bloody stools, and hemoglobinuria. A blood transfusion reaction was suspected and given minor crossmatch positive, T-cryptantigen (TCA) activation was considered likely. The peripheral blood smear reported anemia with moderate anisocytosis, nucleated RBC, and increased spherocytes and schistocytes. Plasma free hemoglobin was elevated to 2280 $\mathrm{mg} / \mathrm{dL}$. She also developed thrombocytopenia and coagulopathy. The patient received blood products that were less likely to exacerbate hemolysis: washed $\mathrm{RBC}$ and platelets, prothrombin complex concentrate (PCC), and fibrinogen concentrate with improvement in coagulopathy.

The clinical course worsened with multiorgan failure and she underwent an exploratory laparotomy where a large sigmoid perforation was found. Over the next two days, the baby underwent two additional explorations of the abdomen and entire small bowel and colon were found to be ischemic, with multiple focal areas of necrosis. After discussions with the family, the care was redirected.

The autopsy's report was consistent with necrotizing enterocolitis totalis. Additionally, multiorgan microvascular fibrin thrombi involving heart, renal glomeruli, stomach, small and large bowel may have been associated with intravascular hemolysis.

\section{Case 2}


A 27 weeks preterm boy was born by cesarean section due to reversed end-diastolic flow and severe intrauterine growth restriction (IUGR). Pregnancy was complicated by maternal history of systemic lupus erythematosus, Chron's disease, and asymptomatic maternal SARS-CoV-2 infection two months before delivery. On DOL 1, he was found to be pancytopenic, followed on DOL 2 by mild disseminated intravascular coagulation (DIC), which responded to plasma, RBC, and platelet transfusions. The patient had intravascular hemolysis with a hemoglobin of $7.2 \mathrm{~g} / \mathrm{dL}$, elevated unconjugated bilirubin $(7 \mathrm{mg} / \mathrm{dL})$, lactate dehydrogenase $(\mathrm{LDH})$ of $555 \mathrm{U} / \mathrm{L}$, high reticulocyte count, and plasma free hemoglobin $(700 \mathrm{mg} / \mathrm{dL})$; the peripheral smear reported nucleated $\mathrm{RBC}$ and schistocytes. The lupus anticoagulant panel was negative. Head ultrasound reported no intraventricular hemorrhage. SARS-CoV-2 antibodies were reactive (Table 2).

\section{Case 3}

A 36 weeks preterm boy was delivered by cesarean section due to fetal decelerations. Pregnancy was complicated by SARS-CoV-2 infection during the second trimester, type 2 diabetes mellitus, hypertension, oligohydramnios, and chorioamniotic separation. At 24 hours of life, he was found to have elevated total bilirubin of $13.6 \mathrm{mg} / \mathrm{dL}$ requiring phototherapy and close to the threshold needing exchange transfusion. Additional investigations were consistent with intravascular hemolysis with elevated reticulocyte count, elevated immature reticulocyte fraction, elevated free plasma hemoglobin $(120 \mathrm{mg} / \mathrm{dL})$, elevated LDH $(1853 \mathrm{U} / \mathrm{L})$, low haptoglobin $(<8 \mathrm{mg} / \mathrm{dL})$. SARS-CoV-2 antibodies were reactive (Table 2). Hyperbilirubinemia responded to intensive phototherapy. Glucose-6 phosphate dehydrogenase (G6PD) level was low at 5.4 units/g Hb.

\section{Discussion}

We describe the clinical course of three neonates born to mothers with history of SARS-CoV-2 infection during pregnancy, who developed intravascular hemolysis while having other concurrent illnesses. Two cases had a reactive anti-SARS-CoV-2 antibodies test. Case 1 had two negative nasopharyngeal swabs for SARSCoV-2 PCR; cases 2 and 3 were not tested given the negative maternal tests at the delivery. Vertical transmission of SARS-CoV-2, although rare, has been reported, but our cases did not meet the criteria for congenital infection. ${ }^{1}$ However, there have been reports of newborns born to SARS-CoV-2 positive mother who presented with thrombocytopenia, refractory shock, and multiorgan failure. ${ }^{2}$ Another study reported NEC in a preterm infant born to a SARS-CoV-2 positive mother. ${ }^{3}$

All our cases developed hemolytic anemia early in their course, and it was associated with conditions that predispose to hemolysis such as NEC, G6PD deficiency, and DIC.

Our first case had NEC, a complication of prematurity with an incidence of 5-10\% and with mortality rates as high as 30\%. ${ }^{45}$ Anemia is well described in NEC and is multi-factorial due to bleeding, iatrogenic losses, thrombotic microangiopathy, or activation of the Thomsen-Friedenrich cryptantigen on the RBC. As many as $30 \%$ of newborns with NEC could have TCA activation. ${ }^{6}$ Surface TCA is an antigen concealed by a layer of N-acetylneuraminic acid. ${ }^{8}$ When TCA is exposed may interact with immunoglobulin M (IgM) anti - T antibodies present in plasma. ${ }^{7}$ When RBCs are destroyed, hemoglobin is released in plasma and couples irreversibly with haptoglobin. This complex is cleared from circulation by monocytes and macrophages. Low levels of haptoglobin, as in cases 2 and 3, are useful to support the hemolytic etiology, but it is important to note that solely low haptoglobin levels in newborns may not be useful for diagnosis of hemolysis. ${ }^{8}$ In case 1 , there was a strong clinical suspicion that TCA activation was responsible for the hemolytic process based on positive minor crossmatch. The patient received washed RBC and platelets, PCC, and fibrinogen concentrate with subsequent improvement in hemolysis. Case 3 had decreased level of G6PD. G6PD deficiency is mostly asymptomatic unless there is exposure to oxidative stress, caused by infections, or medications, followed by hemolysis. ${ }^{8}$ There are reports of a possible association of SARS-CoV2 infection with hemolysis in adults with G6PD deficiency. 910

All cases had elevated free plasma hemoglobin, elevated reticulocyte count, schistocytes on peripheral smear, but the direct antiglobulin test (DAT) was negative. 5-10\% of hemolytic anemias may have negative antibody testing on routine testing, ${ }^{12}$ due to the presence of a different type of antibody (IgM), small number of RBCs bound to IgG molecules or low-affinity autoantibodies. Additionally, during SARS-CoV-2 infection, 
hemolytic anemia may be DAT negative, and traditional hemolytic markers may be unreliable. ${ }^{11}$ As seen in our cases, high plasma hemoglobin levels can be highly pathogenic through direct endothelial damage, lipid peroxidation, or activation of various inflammatory pathways. ${ }^{1121}$

None of the previous reports involving newborns born to SARS-CoV-2 positive mothers describe hemolytic anemia, but there are reports of autoimmune hemolytic anemia in adults with SARS-CoV-2 infection, presumably caused by the cytokine storm or through molecular mimicry. ${ }^{14-17}$ Most of the reported cases of SARS-CoV-2 infection associated with hemolytic anemia had an underlying condition predisposing to hemolysis such as spherocytosis, G-6-PD deficiency, or malignancy ${ }^{18}$ similar to our cases.

Our cases highlight the need for a prospective study to identify if the incidence of hemolysis in neonates born to mothers with active or resolved SARS-CoV-2 infection during pregnancy is indeed increased. While a direct causal relationship between intrauterine exposure to SARS-CoV-2 infection or antibodies and hemolysis cannot be definitively proven, based on the clinical presentation, we speculate that infants with conditions known to cause hemolysis are at increased risk of developing a more severe form of hemolytic anemia. If intravascular hemolysis and coagulopathy are present, until further information is available, using washed blood products that are less likely to exacerbate the hemolytic process, irradiated RBC, and PCC might be a consideration.

\section{Contribution Statement}

Dr. Itriago conceptualized the manuscript, drafted the initial manuscript, and reviewed and revised the manuscript.

Dr. Lingappan, Dr. Teruya and Dr. Dinu conceptualized the manuscript, reviewed and revised the manuscript.

All authors approved the final manuscript as submitted and agree to be accountable for all aspects of the work.

\section{References:}

1. Deniz M, Tezer H. Vertical transmission of SARS CoV-2: a systematic review Melis Deniz \& Hasan Tezer Vertical transmission of SARS CoV-2: a systematic review. doi:10.1080/14767058.2020.1793322

2. Zhu H, Wang L, Fang C, et al. Clinical analysis of 10 neonates born to mothers with 2019-nCoV pneumonia. Transl Pediatr . 2020;9(1):51-60. doi:10.21037/tp.2020.02.06

3. Liu P, Zheng J, Yang P, et al. The immunologic status of newborns born to SARS-CoV-2-infected mothers in Wuhan, China. J Allergy Clin Immunol . 2020;146(1):101. doi:10.1016/j.jaci.2020.04.038

4. Samuels N, van de Graaf RA, de Jonge RCJ, Reiss IKM, Vermeulen MJ. Risk factors for necrotizing enterocolitis in neonates: a systematic review of prognostic studies. BMC Pediatr . 2017;17(1):105. doi:10.1186/s12887-017-0847-3

5. Stout G, Lambert DK, Baer VL, et al. Necrotizing enterocolitis during the first week of life: A multicentered case - Control and cohort comparison study. J Perinatol . 2008;28(8):556-560. doi:10.1038/jp.2008.36

6. Klein RL, Novak RW, Novak PE. T-cryptantigen exposure in neonatal necrotizing enterocolitis. J Pediatr Surg . 1986;21(12):1155-1158. doi:10.1016/0022-3468(86)90031-X

7. Boralessa $\mathrm{H}$, Modi $\mathrm{N}$, Cockburn H, et al. I M M U O H E M A T O L O G Y RBC T Activation and Hemolysis in a Neonatal Intensive Care Population: Implications for Transfusion Practice.

8. Nkhoma ET, Poole C, Vannappagari V, Hall SA, Beutler E. The global prevalence of glucose-6-phosphate dehydrogenase deficiency: A systematic review and meta-analysis. Blood Cells, Mol Dis . 2009;42(3):267278. doi:10.1016/j.bcmd.2008.12.005 
9. Kuipers MT, van Zwieten R, Heijmans J, et al. Glucose-6-phosphate dehydrogenase deficiency-associated hemolysis and methemoglobinemia in a COVID-19 patient treated with chloroquine. Am J Hematol . 2020;95(8):E194-E196. doi:10.1002/ajh.25862

10. Maillart E, Leemans S, Van Noten H, et al. A case report of serious haemolysis in a glucose-6phosphate dehydrogenase-deficient COVID-19 patient receiving hydroxychloroquine. Infect Dis (Auckl) . 2020;52(9):659-661. doi:10.1080/23744235.2020.1774644

11. Lancman G, Marcellino BK, Thibaud S, Troy K. Coombs-negative hemolytic anemia and elevated plasma hemoglobin levels in COVID-19. Ann Hematol . 2020:1. doi:10.1007/s00277-020-04202-3

12. Garratty G. Immune hemolytic anemia associated with negative routine serology. Semin Hematol . 2005;42(3):156-164. doi:10.1053/j.seminhematol.2005.04.005

13. Zanella A, Barcellini W. Treatment of autoimmune hemolytic anemias. Haematologica . 2014;99(10):15471554. doi:10.3324/haematol.2014.114561

14. Angileri F, Légaré S, Marino Gammazza A, Conway de Macario E, Macario AJL, Cappello F. Is molecular mimicry the culprit in the autoimmune haemolytic anaemia affecting patients with COVID-19? Br J Haematol . 2020;190(2):e92-e93. doi:10.1111/bjh.16883

15. Lazarian G, Quinquenel A, Bellal M, et al. Autoimmune haemolytic anaemia associated with COVID-19 infection. Br J Haematol . 2020;190(1):29-31. doi:10.1111/bjh.16794

16. Wahlster L, Weichert-Leahey N, Trissal M, Grace RF, Sankaran VG. COVID-19 presenting with autoimmune hemolytic anemia in the setting of underlying immune dysregulation. Pediatr Blood Cancer . 2020;67(9). doi:10.1002/pbc.28382

17. Hindilerden F, Yonal-Hindilerden I, Akar E, Yesilbag Z, Kart-Yasar K. Severe autoimmune hemolytic anemia in Covid-19 infection.Mediterr J Hematol Infect Dis . 2020;12(1):e2020053. doi:10.4084/mjhid.2020.053

18. Severance TS, Rahim MQ, French J, et al. COVID-19 and hereditary spherocytosis: A recipe for hemolysis. Pediatr Blood Cancer. July 2020. doi:10.1002/pbc.28548

\section{Hosted file}

Table 1.pdf available at https://authorea.com/users/370192/articles/488895-case-seriesof-hemolysis-in-neonate-born-to-mothers-with-history-of-sars-cov-2-infection-duringpregnancy

\section{Hosted file}

Table 2.pdf available at https://authorea.com/users/370192/articles/488895-case-seriesof-hemolysis-in-neonate-born-to-mothers-with-history-of-sars-cov-2-infection-duringpregnancy 